\title{
Experimental Design in reservoir simulation: an integrated solution for uncertainty analysis, a case study
}

\author{
Fatemeh Moeinikia • Nasser Alizadeh
}

Received: 20 November 2011/Accepted: 17 April 2012/Published online: 3 May 2012

(C) The Author(s) 2012. This article is published with open access at Springerlink.com

\begin{abstract}
Quantification of uncertain parameters in oil reservoirs is one of the major issues of concern. In underdeveloped reservoirs, there are many uncertain parameters affecting production forecast which plays a main role in reservoir management and decision making in development plan. To study the effect of uncertain parameters on the behavior of a reservoir and to forecast the probabilistic production of the reservoir, the simulator has to be run too many times with different entries for uncertain parameters. To avoid this heavy and timeconsuming process, Experimental Design methodology is used which chooses the values of uncertain parameters from their ranges in a way that the total uncertainty in the system is captured with the least number of simulator runs. In this study, Experimental Design methodology is used to observe the effect of uncertain parameters on the production of an underdeveloped oil reservoir, which is subjected to immiscible gas injection method, and to estimate the probabilistic production of the reservoir; therefore, the proper and unbiased decisions for oil reservoir development can be made. Experimental Design methodology, as a powerful and trusted method, makes it possible to choose simulator runs so as to obtain accurate probabilistic production diagrams using the least number of runs as well as to study the impact of uncertain parameters on the oil reservoir production profile, quickly.
\end{abstract}

Keywords Uncertainty analysis - Experimental Design · Sensitivity analysis · Reservoir simulation - Immiscible gas injection

F. Moeinikia $(\bowtie) \cdot$ N. Alizadeh

Petroleum Engineering Department,

Amirkabir University of Technology, Tehran, Iran

e-mail: moinikia@aut.ac.ir

$\begin{array}{ll}\text { List of symbols } \\ \text { COP } & \text { Cumulative oil production } \\ \text { GU } & \text { General uncertainty } \\ \text { MULTPV } & \text { Pore volume multiplier } \\ \text { MULTX } & \text { Transmissibility multiplier in } X \text { direction } \\ \text { MULTY } & \text { Transmissibility multiplier in } Y \text { direction } \\ \text { MULTZ } & \text { Transmissibility multiplier in } Z \text { direction } \\ \text { MULTFLT } & \text { Transmissibility multiplier for fault } \\ \text { BHPinj } & \text { Bottom hole pressure of injection well } \\ \text { Qinj } & \text { Surface injection rate } \\ \text { Pia } & \text { Aquifer productivity index } \\ \text { Va } & \text { Initial volume of water in aquifer } \\ \text { SGCR } & \text { Critical gas saturation } \\ \text { OIP } & \text { Oil in place } \\ \text { PV } & \text { Pore volume } \\ S_{\mathrm{o}} & \text { Oil saturation } \\ S_{\mathrm{w}} & \text { Water saturation } \\ S_{\mathrm{g}} & \text { Gas saturation } \\ B_{\mathrm{o}} & \text { Oil formation volume factor } \\ \text { DC } & \text { Production due to rock compaction } \\ \text { DW } & \text { Production due to water influx } \\ \text { DG } & \text { Production due to gas influx } \\ \text { DE } & \text { Production due to oil expansion } \\ \text { DS } & \text { Production due to solution gas } \\ & \end{array}$

\section{Introduction}

In recent years, immiscible gas injection, as an effective method for enhanced oil recovery, has been used in many oil reservoirs. To optimize the development plan for an underdeveloped reservoir associated with many uncertain parameters and subjected to this method of enhanced oil recovery, it is necessary to determine the effect of uncertain parameters on the behavior of the oil reservoir.

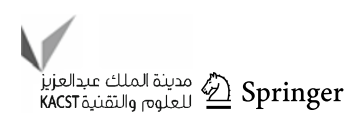


Studying all of these uncertain parameters is an inefficient and time-consuming process. The main idea in this study is to find the major uncertain parameters with the most significant effect on the reservoir behavior.

To study the effect of these main uncertain parameters as well as to acquire the probabilistic production forecast, it is a good idea to use effective simulator runs to trap the whole uncertainty instead of running the simulator thousands of times with diverse entries for uncertain parameters.

Experimental Design methodology is able to select the effective uncertain parameters and to study their effect on the reservoir production with the minimum number of simulator runs.

Experimental Design methodology has already been applied in petroleum industry. For example, Experimental Design has been used to develop a polynomial model. This model has been then used to analyze the uncertainty available in the reservoir (Damsleth et al. 1991; Venkataraman 2000; Kabir et al. 2002) and also used for optimization (Dejean and Blanc 1999; White and Royer 2003; Zhang et al. 2007). In addition, Experimental Design has been combined with response surface to assess uncertainty in reservoir (Fetel and Caumon 2008). Moreover, Experimental Design has been used to eliminate options which impacted negatively on project economics and to select those that added net present value and to optimize development plan of reservoir (Kloosterman et al. 2007, 2008). In one research, a response surface was generated using Experimental Design. This response surface was used for improving development plan of agbami field (Spokes et al. 2004). In another research, Experimental Design was used to assess the uncertainty in a carbonate oil reservoir under water injection (Tabari 2010).

In this study, Experimental Design methodology is directly applied for uncertainty investigation in an underdeveloped oil reservoir which is subjected to immiscible gas injection method to take a step towards developing this reservoir.

\section{Experimental Design methodology}

Experimental Design method distributes the simulation runs within uncertain ranges of parameters efficiently, thereby minimizes the number of required runs for studying an uncertain system (Steppan et al. 1998). In fact, this methodology provides a quantified decision-based plan for minimizing risk in oil reservoirs (Kloosterman et al. 2008).

In this study, one variable at a time design is used for choosing the most effective uncertain parameters on the reservoir behavior. Furthermore, the inscribed central composite design and three-level full factorial design are combined to study the impact of effective uncertain parameters on reservoir production forecast.

\section{Dena field specifications}

This study has been performed on an underdeveloped oil reservoir, subjected to immiscible gas injection method, and situated in the southern part of Iran (Fig. 1). The specifications of this reservoir are presented in Table 1.

\section{Sensitivity analysis}

Many uncertain parameters are present in the simulation of Dena reservoir. The corresponding Ranges of these parameters, obtained from analogous reservoirs, are presented in Table 2.

In this stage, one variable at a time design is used to choose the uncertain parameters with the most significant effect on the specified outcome (herein, COP). The present study uses this design with a slight difference: instead of medium entries, base entries are used. Moreover, the case with all parameters at medium entries will be omitted from this design.

Various entries for uncertain parameters, which have been chosen according to this design, and forecasted COP values by the simulator, are presented in Table 3 .

According to Figs. 2, 3 and 4, which are the results of the sensitivity analysis, it is clear that MULTPV, MULTZ, Qinj and SGCR parameters have the most significant effect on the COP in the 4th, 8th and 12th years from the beginning of simulation in Dena reservoir. The method for calculating the effect of uncertain parameters on the COP has been explained in Appendix. These effective parameters will be studied in the next stages.

\section{Uncertainty analysis}

Dena field is currently under development. The immiscible gas injection for pressure maintenance is considered for this field. A better understanding of the reservoir behavior with respect to the existing effective uncertain parameters and minimizing the effect of this uncertainty on production profile can help to improve Dena field development plan.

The objective in this stage is to study the impact of effective uncertain parameters, chosen in the previous stage, on the reservoir behavior and to acquire the probabilistic production diagrams for Dena reservoir. By integrating three-level full factorial and inscribed central composite designs, the entries for effective uncertain parameters from their ranges are chosen. The method of 
Fig. 1 Four views of Dena reservoir
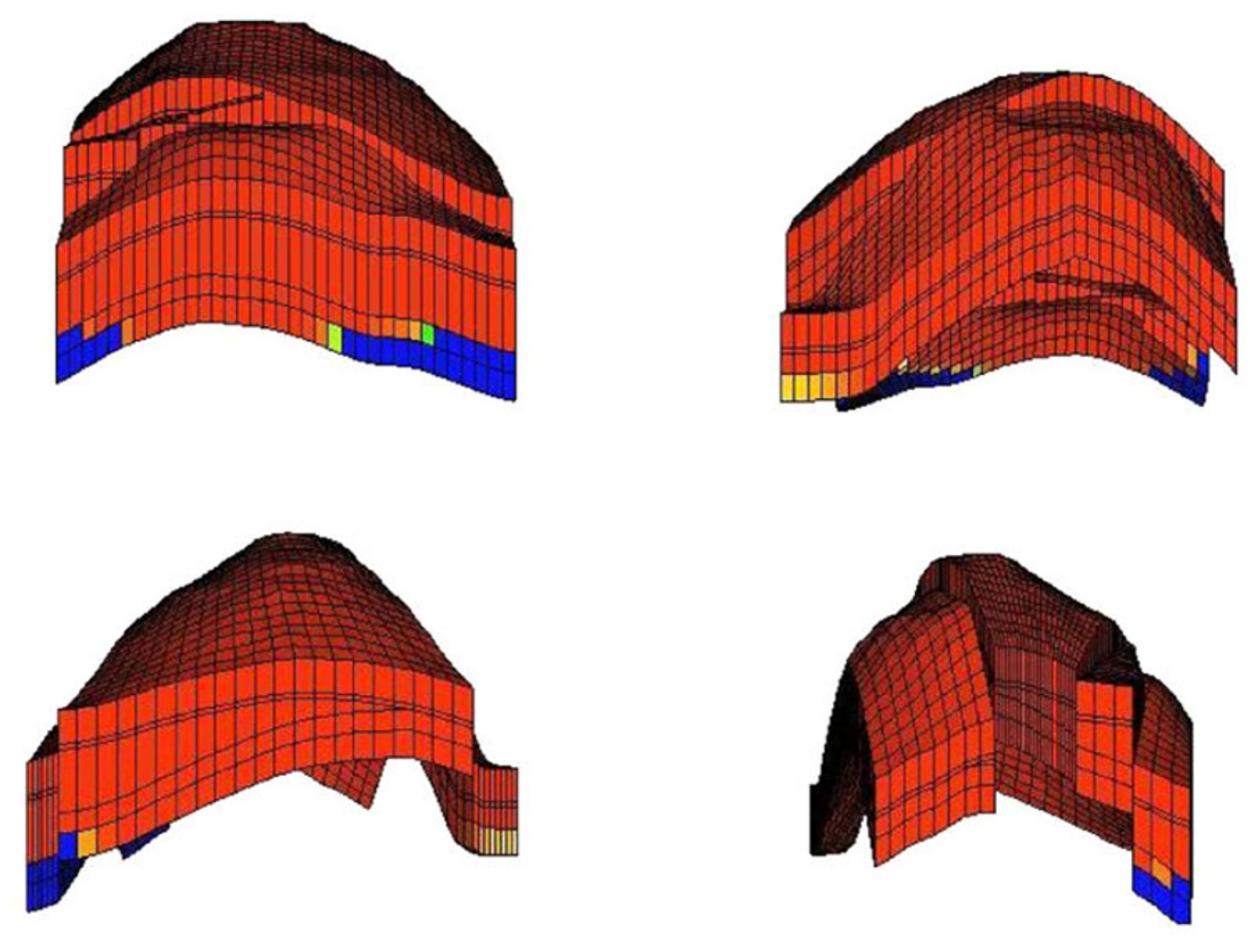

Oilsiat

$\begin{array}{lllll}0.00000 & 0.20851 & 0.41702 & 0.62554 & 0.834-0.5\end{array}$

Table 1 Characteristics of Dena field

\begin{tabular}{ll}
\hline Parameter & $\begin{array}{l}\text { Value of the } \\
\text { parameter }\end{array}$ \\
\hline Location of the field & $\begin{array}{c}\text { Southern part } \\
\text { of Iran }\end{array}$ \\
Area of the field $\left(\mathrm{km}^{2}\right)$ & 210 \\
Gross thickness of the formation (m) & 182 \\
Average porosity of the formation (\%) & 27 \\
Average permeability of the formation & 14 \\
in $X$ direction (mD) & 14 \\
Average permeability of the formation & \\
in $Y$ direction (mD) & 71 \\
Average permeability of the formation & \\
in $Z$ direction (mD) & 20 \\
Low water saturation (\%) & 12 \\
Number of wells for oil production & 1 \\
Number of wells for gas injection &
\end{tabular}

choosing uncertain parameters values based on integrating these two designs is presented in Tables 4 and 5. Subsequently, the simulator is run to calculate COP values corresponding to the chosen entries. In this study, Eclipse 100 has been employed as the simulator. Eclipse 100 calculates COP taking into consideration that the total oil produced equals the change in oil-in-place. These calculations have been explained in Eqs. 1 to 7 (Eclipse 2005).
Table 2 Minimum, base and maximum values for uncertain parameters existing in the simulation of Dena reservoir

\begin{tabular}{llll}
\hline $\begin{array}{l}\text { Uncertain } \\
\text { parameter }\end{array}$ & $\begin{array}{l}\text { Minimum } \\
\text { value }\end{array}$ & $\begin{array}{l}\text { Base } \\
\text { value }\end{array}$ & $\begin{array}{l}\text { Maximum } \\
\text { value }\end{array}$ \\
\hline MULTPV & 0.5 & 1 & 2 \\
MULTX & 0.5 & 1 & 2 \\
MULTY & 0.5 & 1 & 2 \\
MULTZ & 0.5 & 1 & 2 \\
MULTFLT & 0.5 & 1 & 2 \\
BHPinj (Psia) & 4,000 & 5,000 & 6,000 \\
Qinj (Mscf/day) & 1,000 & 5,000 & 10,000 \\
Pia (STB/day/Psi) & 10 & 200 & 400 \\
Va (STB) & $7 \mathrm{E}+07$ & $7 \mathrm{E}+08$ & $7 \mathrm{E}+09$ \\
SGCR & 0.1 & 0.2 & 0.3 \\
\hline
\end{tabular}

$\mathrm{d}(\mathrm{OIP})=\mathrm{PV}^{t+\mathrm{d} t} S_{\mathrm{o}}^{t+\mathrm{d} t}\left[1 / B_{o}\right]^{t+\mathrm{d} t}-\mathrm{PV}^{t} S_{\mathrm{o}}^{t}\left[1 / B_{\mathrm{o}}\right]^{t}$,

where

$$
\begin{aligned}
\mathrm{PV}^{t+\mathrm{d} t} & =\mathrm{PV}^{t}+\mathrm{d}(\mathrm{PV}) \\
S_{\mathrm{o}}^{t+\mathrm{d} t} & =S_{\mathrm{o}}^{t}+\mathrm{d}\left(S_{\mathrm{o}}\right) \\
{\left[1 / B_{o}\right]^{t+\mathrm{d} t} } & =\left[1 / B_{\mathrm{o}}\right]^{t}+\mathrm{d}\left[1 / B_{\mathrm{o}}\right]
\end{aligned}
$$

Substituting this equation into Eq. 1, we have

$$
\begin{aligned}
\mathrm{d}(\mathrm{OIP})= & {[\mathrm{PV}+\mathrm{d}(\mathrm{PV})]\left[S_{\mathrm{o}}+\mathrm{d}\left(S_{\mathrm{o}}\right)\right]\left[\left(1 / B_{\mathrm{o}}\right)+\mathrm{d}\left(1 / B_{\mathrm{o}}\right)\right] } \\
& -\left[\mathrm{PV} S_{\mathrm{o}}\left(1 / B_{\mathrm{o}}\right)\right]
\end{aligned}
$$


Table 3 Values of COP, calculated by simulator, corresponding to various values of uncertain parameters which are selected based on one variable at a time design

\begin{tabular}{|c|c|c|c|c|c|c|c|c|c|c|c|c|}
\hline MULTPV & MULTX & MULTY & MULTZ & MULTFLT & BHPinj & Qinj & Pia & $\mathrm{Va}$ & SGCR & $\begin{array}{l}\text { COP after } \\
4 \text { years }(\mathrm{STB})\end{array}$ & $\begin{array}{l}\text { COP after } \\
8 \text { years }(\mathrm{STB})\end{array}$ & $\begin{array}{l}\text { COP after } \\
12 \text { years }(\mathrm{STB})\end{array}$ \\
\hline+1 & 0 & 0 & 0 & 0 & 0 & 0 & 0 & 0 & 0 & 15898508 & 33090864 & 47734612 \\
\hline-1 & 0 & 0 & 0 & 0 & 0 & 0 & 0 & 0 & 0 & 12799578 & 24386626 & 33867964 \\
\hline 0 & +1 & 0 & 0 & 0 & 0 & 0 & 0 & 0 & 0 & 14131435 & 29233024 & 41012624 \\
\hline 0 & -1 & 0 & 0 & 0 & 0 & 0 & 0 & 0 & 0 & 13992454 & 28839466 & 39969692 \\
\hline 0 & 0 & +1 & 0 & 0 & 0 & 0 & 0 & 0 & 0 & 14148386 & 28264708 & 41256968 \\
\hline 0 & 0 & -1 & 0 & 0 & 0 & 0 & 0 & 0 & 0 & 13993905 & 27823774 & 39882720 \\
\hline 0 & 0 & 0 & +1 & 0 & 0 & 0 & 0 & 0 & 0 & 15375486 & 31294306 & 44569036 \\
\hline 0 & 0 & 0 & -1 & 0 & 0 & 0 & 0 & 0 & 0 & 13510327 & 26250094 & 36799040 \\
\hline 0 & 0 & 0 & 0 & +1 & 0 & 0 & 0 & 0 & 0 & 14599191 & 29001026 & 40869236 \\
\hline 0 & 0 & 0 & 0 & -1 & 0 & 0 & 0 & 0 & 0 & 14455951 & 28695374 & 40432100 \\
\hline 0 & 0 & 0 & 0 & 0 & +1 & 0 & 0 & 0 & 0 & 14537121 & 28868602 & 40678432 \\
\hline 0 & 0 & 0 & 0 & 0 & -1 & 0 & 0 & 0 & 0 & 14437271 & 28699240 & 40473240 \\
\hline 0 & 0 & 0 & 0 & 0 & 0 & +1 & 0 & 0 & 0 & 14913045 & 30525334 & 43463004 \\
\hline 0 & 0 & 0 & 0 & 0 & 0 & -1 & 0 & 0 & 0 & 14267080 & 27781618 & 38541204 \\
\hline 0 & 0 & 0 & 0 & 0 & 0 & 0 & +1 & 0 & 0 & 14530310 & 28864704 & 40677280 \\
\hline 0 & 0 & 0 & 0 & 0 & 0 & 0 & -1 & 0 & 0 & 14491061 & 28678376 & 40354836 \\
\hline 0 & 0 & 0 & 0 & 0 & 0 & 0 & 0 & +1 & 0 & 14540457 & 28997110 & 41068692 \\
\hline 0 & 0 & 0 & 0 & 0 & 0 & 0 & 0 & -1 & 0 & 14493547 & 28624562 & 40172736 \\
\hline 0 & 0 & 0 & 0 & 0 & 0 & 0 & 0 & 0 & +1 & 14198728 & 28683066 & 40075848 \\
\hline 0 & 0 & 0 & 0 & 0 & 0 & 0 & 0 & 0 & -1 & 14761498 & 30615244 & 44939368 \\
\hline
\end{tabular}

$-1,+1,0$ represent minimum, maximum and base values, respectively

Expanding the Eq. 3,

$$
\begin{aligned}
\mathrm{d}(\mathrm{OIP})= & \mathrm{d}(\mathrm{PV}) S_{\mathrm{o}}^{t}\left[1 / B_{\mathrm{o}}\right]^{t}+\mathrm{d}(\mathrm{PV}) S_{\mathrm{o}}^{t} d\left[1 / B_{\mathrm{o}}\right] \\
& +\mathrm{PV}^{t} \mathrm{~d}\left(S_{\mathrm{o}}\right)\left[1 / B_{\mathrm{o}}\right]^{t}+\mathrm{d}(\mathrm{PV}) \mathrm{d}\left(S_{\mathrm{o}}\right)\left[1 / B_{\mathrm{o}}\right]^{t} \\
& +\mathrm{PV}^{t} S_{\mathrm{o}}^{t} \mathrm{~d}\left[1 / B_{\mathrm{o}}\right]+\mathrm{PV}^{t} \mathrm{~d}\left(S_{\mathrm{o}}\right) \mathrm{d}\left[1 / B_{\mathrm{o}}\right] \\
& +\mathrm{d}(\mathrm{PV}) \mathrm{d}\left(S_{\mathrm{o}}\right) \mathrm{d}\left[1 / B_{\mathrm{o}}\right]
\end{aligned}
$$

The terms in this equation are associated with the various production mechanisms. These mechanisms are introduced in the following:

$$
\begin{aligned}
& \mathrm{DC}=-\mathrm{d}(\mathrm{PV}) S_{\mathrm{o}}^{t}\left[1 / B_{\mathrm{o}}\right]^{t+\mathrm{d} t} \\
& \mathrm{DS}=-\mathrm{PV}^{t+\mathrm{d} t} \mathrm{~d}\left(S_{\mathrm{o}}\right)\left[1 / B_{\mathrm{o}}\right]^{t} \\
& \mathrm{DE}=-\mathrm{PV}^{t} S_{\mathrm{o}}^{t+\mathrm{d} t} \mathrm{~d}\left[1 / B_{\mathrm{o}}\right]-\mathrm{d}(\mathrm{PV}) \mathrm{d}\left(S_{\mathrm{o}}\right) \mathrm{d}\left[1 / B_{\mathrm{o}}\right]
\end{aligned}
$$

Now, we know that

$\mathrm{d}\left(S_{\mathrm{o}}\right)=-\mathrm{d}\left(S_{\mathrm{w}}\right)-\mathrm{d}\left(S_{\mathrm{g}}\right)$

Therefore,

$$
\begin{aligned}
\mathrm{DC} & =-\mathrm{d}(\mathrm{PV}) S_{\mathrm{o}}^{t}\left[1 / B_{\mathrm{o}}\right]^{t+\mathrm{d} t} \\
\mathrm{DW} & =\mathrm{PV}^{t+\mathrm{d} t} \mathrm{~d}\left(S_{w}\right)\left[1 / B_{\mathrm{o}}\right]^{t} \\
\mathrm{DG} & =\mathrm{PV}^{t+\mathrm{d} t} \mathrm{~d}\left(S_{\mathrm{g}}\right)\left[1 / B_{\mathrm{o}}\right]^{t} \\
\mathrm{DE} & =-\mathrm{PV}^{t} S_{\mathrm{o}}^{t+\mathrm{d} t} \mathrm{~d}\left[1 / B_{\mathrm{o}}\right]-\mathrm{d}(\mathrm{PV}) \mathrm{d}\left(S_{\mathrm{o}}\right) \mathrm{d}\left[1 / B_{\mathrm{o}}\right]
\end{aligned}
$$

Now, using the simulator outcomes, the probabilistic production diagrams of Dena reservoir for the 4th, 8 th and 12 th years from the beginning of the simulation will be forecasted (Figs. 5, 6, 7).

According to Figs. 5, 6 and 7, the uncertainty for COP is very high and the forecasted COP values vary from 11439804 STB to 17282130 STB for the 4th year, 19941184 STB to 36872300 STB for the 8th year and 25958506 STB to 54636132 STB for the 12th year from the beginning of simulation. This is due to the uncertainty, present in the reservoir.

According to Figs. 5, 6 and 7, GU is calculated using the following relationship (Dejean J and Blanc 1999):

$$
\mathrm{GU}=\frac{\mathrm{COP}(\max )-\operatorname{COP}(\min )}{(\operatorname{COP}(\max )+\operatorname{COP}(\min )) / 2}
$$

Therefore, GUs at the 4th, 8th and 12th years from the beginning of the simulation are 41, 60 and $71 \%$, respectively, which are all high values.

The maximum value for COP at the 12th year is related to the maximum values for MULTPV, MULTZ, Qinj and the minimum value for SGCR from their ranges.

Since MULTPV, MULTZ and SGCR parameters are uncontrollable, the medium values of these parameters are 


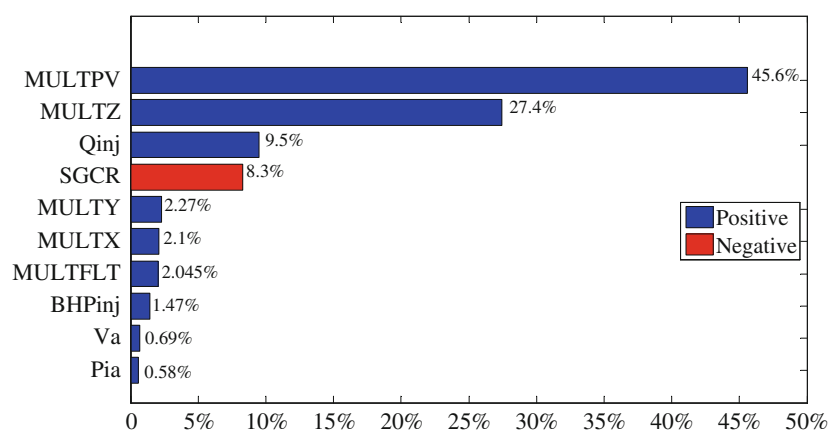

Fig. 2 Sensitivity study showing the effect of uncertain parameters on COP after 4 years from the beginning of simulation

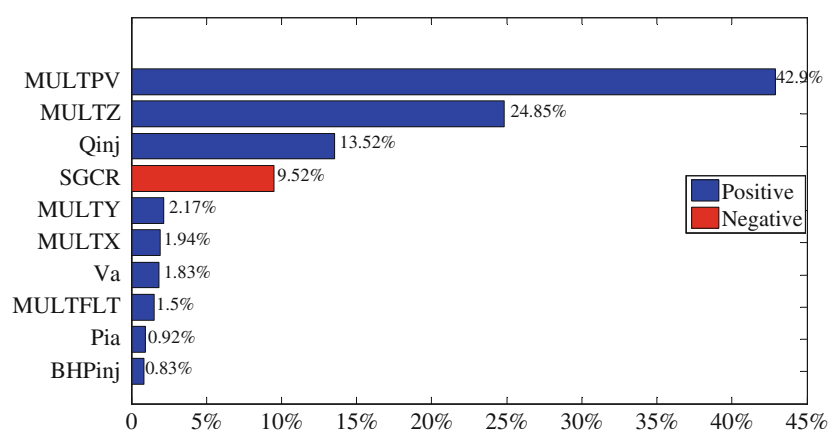

Fig. 3 Sensitivity study showing the effect of uncertain parameters on COP after 8 years from the beginning of simulation

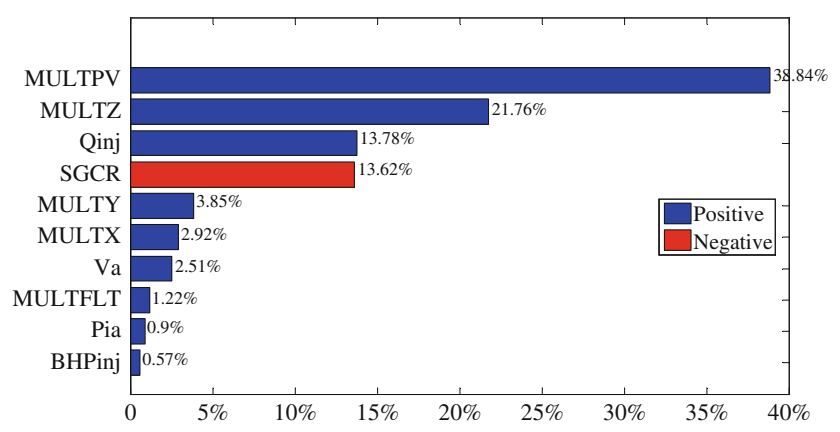

Fig. 4 Sensitivity study showing the effect of uncertain parameters on $\mathrm{COP}$ after 12 years from the beginning of simulation

used and the effect of different Qinj values on the COP will be examined.

According to Fig. 8, as expected, increase in the value of Qinj is associated with increase in oil production, so that the maximum value of $\mathrm{COP}$ will be acquired at Qinj $=10,000 \mathrm{Mscf} /$ day.

In another research (Moeinikia and Alizadeh 2011), a proxy model has been developed by artificial neural network in Dena reservoir. This artificial neural network was run 20,000 times to acquire the entire range of probabilistic production forecast. According to those diagrams, it was concluded that COP values vary from $1.1534 \mathrm{e}+007 \mathrm{STB}$ to $1.7484 \mathrm{e}+007 \mathrm{STB}$ for the 4 th year, $2.1080 \mathrm{e}+007 \mathrm{STB}$ to $3.7598 \mathrm{e}+007 \mathrm{STB}$ for the 8 th year and $2.6901 \mathrm{e}+007 \mathrm{STB}$ to $6.0888 \mathrm{e}+007 \mathrm{STB}$ for the 12 th year. These results are very close to the ones in this study. It confirms that the probabilistic production diagrams in this study, which have been obtained by simulation runs chosen according to Experimental Design methodology, are valid and accurate and cover the whole range of probabilistic production forecast.

Developing an artificial neural network as a proxy model to acquire these diagrams has difficulties such as training of artificial neural network. In addition, developing other proxy models may cause the results to be far from the simulation ones and this leads to wrong results. On the other hand, running a simulator thousands of times, to acquire these diagrams, is very time consuming and expensive. The results of this study show that by direct employment of Experimental Design methodology, one can get the accurate results with the least number of simulation runs instead of running the simulator numerous times or developing a proxy model.

The Integration of three-level full factorial and inscribed central composite designs, which has been employed in this study, provides three inner points within uncertain ranges of parameters and also includes all combinations of parameters values; therefore, one can be more sure that the whole range and also the interaction of uncertain parameters have been covered compared to the other designs in which just one center point and border points are provided to study.

\section{Results and discussion}

Dena reservoir, which is under immiscible gas injection method, is an underdeveloped reservoir with many uncertain parameters.

The key idea in this study was to use one variable at a time design to choose the most effective uncertain parameters on reservoir behavior. Using this design, MULTPV, MULTZ, Qinj and SGCR parameters are identified as the most effective parameters relating to $\mathrm{COP}$ in this reservoir.

To make efficient decisions over the reservoir development plan, the most effective uncertain parameters on the reservoir behavior must be studied and the probabilistic production of the reservoir has to be forecasted. Experimental Design methodology, as a fast and reliable method for obtaining probabilistic results, seems a proper idea for dealing with uncertainty.

In this study, integrating three-level full factorial and inscribed central composite designs, as well as utilizing a simulator, were used to forecast the probabilistic 
Table 4 Values of uncertain parameters selected based on three-level full factorial design

\begin{tabular}{|c|c|c|c|c|c|c|c|c|c|}
\hline RUN & MULTPV & MULTZ & SGCR & Qinj & RUN & MULTPV & MULTZ & SGCR & Qijn \\
\hline 1 & -1 & -1 & -1 & -1 & 42 & -1 & 0 & -1 & 0 \\
\hline 2 & -1 & -1 & -1 & 1 & 43 & 1 & 0 & -1 & 0 \\
\hline 3 & -1 & -1 & 1 & -1 & 44 & -1 & 0 & 1 & 0 \\
\hline 4 & -1 & -1 & 1 & 1 & 45 & 0 & 1 & 0 & 1 \\
\hline 5 & -1 & 1 & -1 & -1 & 46 & 0 & -1 & 0 & -1 \\
\hline 6 & -1 & 1 & -1 & 1 & 47 & 0 & 1 & 0 & -1 \\
\hline 7 & -1 & 1 & 1 & -1 & 48 & 0 & -1 & 0 & 1 \\
\hline 8 & -1 & 1 & 1 & 1 & 49 & -1 & -1 & -1 & 0 \\
\hline 9 & 1 & -1 & -1 & -1 & 50 & -1 & -1 & 1 & 0 \\
\hline 10 & 1 & -1 & -1 & 1 & 51 & -1 & 1 & -1 & 0 \\
\hline 11 & 1 & -1 & 1 & -1 & 52 & -1 & 1 & 1 & 0 \\
\hline 12 & 1 & -1 & 1 & 1 & 53 & 1 & -1 & -1 & 0 \\
\hline 13 & 1 & 1 & -1 & -1 & 54 & 1 & -1 & 1 & 0 \\
\hline 14 & 1 & 1 & -1 & 1 & 55 & 1 & 1 & -1 & 0 \\
\hline 15 & 1 & 1 & 1 & -1 & 56 & 1 & 1 & 1 & 0 \\
\hline 16 & 1 & 1 & 1 & 1 & 57 & 0 & -1 & -1 & -1 \\
\hline 17 & 1 & 0 & 0 & 0 & 58 & 0 & -1 & -1 & 1 \\
\hline 18 & -1 & 0 & 0 & 0 & 59 & 0 & -1 & 1 & -1 \\
\hline 19 & 0 & 1 & 0 & 0 & 60 & 0 & -1 & 1 & 1 \\
\hline 20 & 0 & -1 & 0 & 0 & 61 & 0 & 1 & -1 & -1 \\
\hline 21 & 0 & 0 & 1 & 0 & 62 & 0 & 1 & -1 & 1 \\
\hline 22 & 0 & 0 & -1 & 0 & 63 & 0 & 1 & 1 & -1 \\
\hline 23 & 0 & 0 & 0 & 1 & 64 & 0 & 1 & 1 & 1 \\
\hline 24 & 0 & 0 & 0 & -1 & 65 & -1 & 0 & -1 & -1 \\
\hline 25 & 1 & 1 & 0 & 0 & 66 & -1 & 0 & -1 & 1 \\
\hline 26 & -1 & -1 & 0 & 0 & 67 & -1 & 0 & 1 & -1 \\
\hline 27 & 1 & -1 & 0 & 0 & 68 & -1 & 0 & 1 & 1 \\
\hline 28 & -1 & 1 & 0 & 0 & 69 & 1 & 0 & -1 & -1 \\
\hline 29 & 0 & 0 & 1 & 1 & 70 & 1 & 0 & -1 & 1 \\
\hline 30 & 0 & 0 & -1 & -1 & 71 & 1 & 0 & 1 & -1 \\
\hline 31 & 0 & 0 & 1 & -1 & 72 & 1 & 0 & 1 & 1 \\
\hline 32 & 0 & 0 & -1 & 1 & 73 & -1 & -1 & 0 & -1 \\
\hline 33 & 1 & 0 & 0 & 1 & 74 & -1 & -1 & 0 & 1 \\
\hline 34 & -1 & 0 & 0 & -1 & 75 & -1 & 1 & 0 & -1 \\
\hline 35 & 1 & 0 & 0 & -1 & 76 & -1 & 1 & 0 & 1 \\
\hline 36 & -1 & 0 & 0 & 1 & 77 & 1 & -1 & 0 & -1 \\
\hline 37 & 0 & 1 & 1 & 0 & 78 & 1 & -1 & 0 & 1 \\
\hline 38 & 0 & -1 & -1 & 0 & 79 & 1 & 1 & 0 & -1 \\
\hline 39 & 0 & 1 & -1 & 0 & 80 & 1 & 1 & 0 & 1 \\
\hline 40 & 0 & -1 & 1 & 0 & 81 & 0 & 0 & 0 & 0 \\
\hline 41 & 1 & 0 & 1 & 0 & & & & & \\
\hline
\end{tabular}

$-1,+1,0$ represent minimum, maximum and medium values, respectively

production of Dena reservoir and also to study the effect of the existing uncertainty in the reservoir on the probabilistic production forecast.
As predicted, the uncertainty on the reservoir COP was very high. Moreover, as time goes by, the uncertainty of COP will increase. 
Table 5 Values of uncertain parameters selected based on inscribed central composite design

\begin{tabular}{|c|c|c|c|c|c|c|c|c|c|}
\hline RUN & MULTPV & MULTZ & SGCR & Qinj & RUN & MULTPV & MULTZ & SGCR & Qijn \\
\hline 1 & -1 & -1 & -1 & -1 & 15 & 1 & 1 & 1 & -1 \\
\hline 2 & -1 & -1 & -1 & 1 & 16 & 1 & 1 & 1 & 1 \\
\hline 3 & -1 & -1 & 1 & -1 & 17 & 2 & 0 & 0 & 0 \\
\hline 4 & -1 & -1 & 1 & 1 & 18 & -2 & 0 & 0 & 0 \\
\hline 5 & -1 & 1 & -1 & -1 & 19 & 0 & 2 & 0 & 0 \\
\hline 6 & -1 & 1 & -1 & 1 & 20 & 0 & -2 & 0 & 0 \\
\hline 7 & -1 & 1 & 1 & -1 & 21 & 0 & 0 & 2 & 0 \\
\hline 8 & -1 & 1 & 1 & 1 & 22 & 0 & 0 & -2 & 0 \\
\hline 9 & 1 & -1 & -1 & -1 & 23 & 0 & 0 & 0 & 2 \\
\hline 10 & 1 & -1 & -1 & 1 & 24 & 0 & 0 & 0 & -2 \\
\hline 11 & 1 & -1 & 1 & -1 & 25 & 0 & 0 & 0 & 0 \\
\hline 12 & 1 & -1 & 1 & 1 & 26 & 0 & 0 & 0 & 0 \\
\hline 13 & 1 & 1 & -1 & -1 & 27 & 0 & 0 & 0 & 0 \\
\hline 14 & 1 & 1 & -1 & 1 & 28 & 0 & 0 & 0 & 0 \\
\hline
\end{tabular}

$\pm 2, \pm 1$ and 0 represent extreme, inner and medium points, respectively

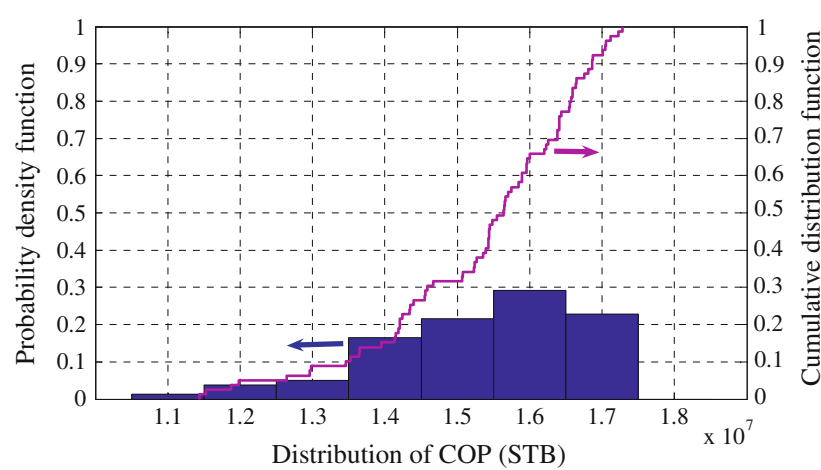

Fig. 5 Probability distribution for COP after 4 years from the beginning of simulation

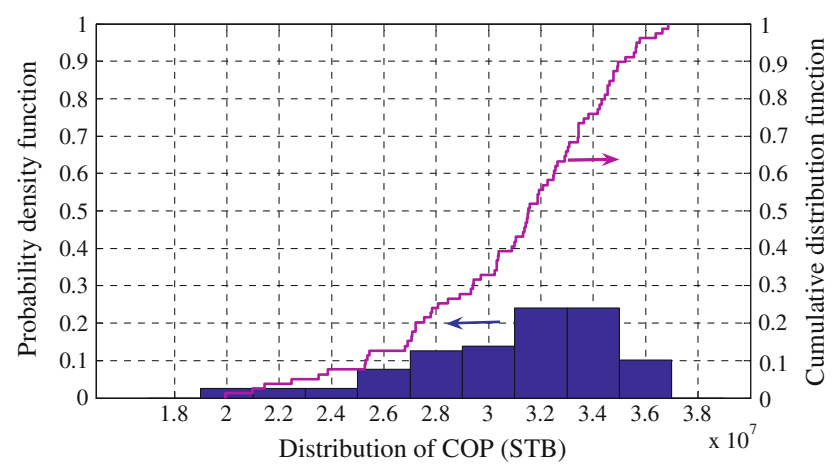

Fig. 6 Probability distribution for COP after 8 years from the beginning of simulation

The results of probabilistic production diagrams in this study were compared to the diagrams, which have been obtained by artificial neural network, and it was observed

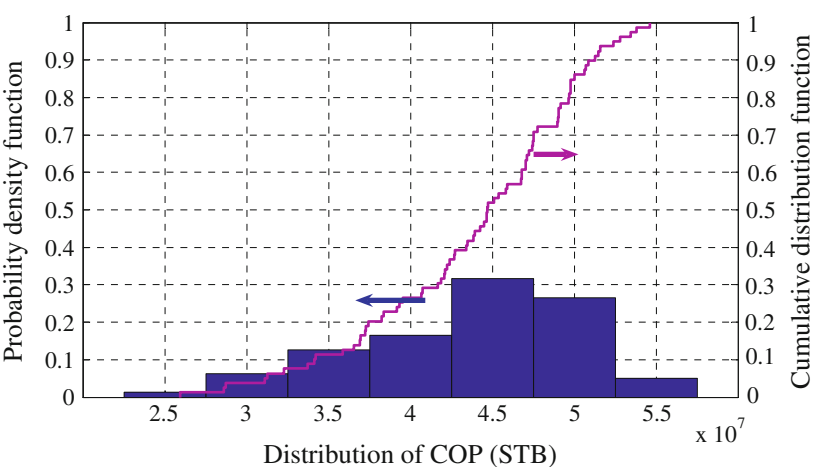

Fig. 7 Probability distribution for COP after 12 years from the beginning of simulation

that these results are nearly the same; therefore, it confirms that direct use of Experimental Design is a trusted and easy method for obtaining the probabilistic production forecast.

\section{Conclusions}

Experimental Design methodology covers the whole uncertainty available in the system with the minimum number of simulator runs. Actually, this method, as an unbiased approach, has the potential of adding useful information to the reservoir development plan and also, of saving considerable time. As a result, it is an efficient method for studying underdeveloped reservoirs.

The main advantage of integrating three-level full factorial and inscribed central composite designs is that many 
Fig. 8 Sensitivity of COP after 12 years from the beginning of simulation with respect to the different values of Qinj

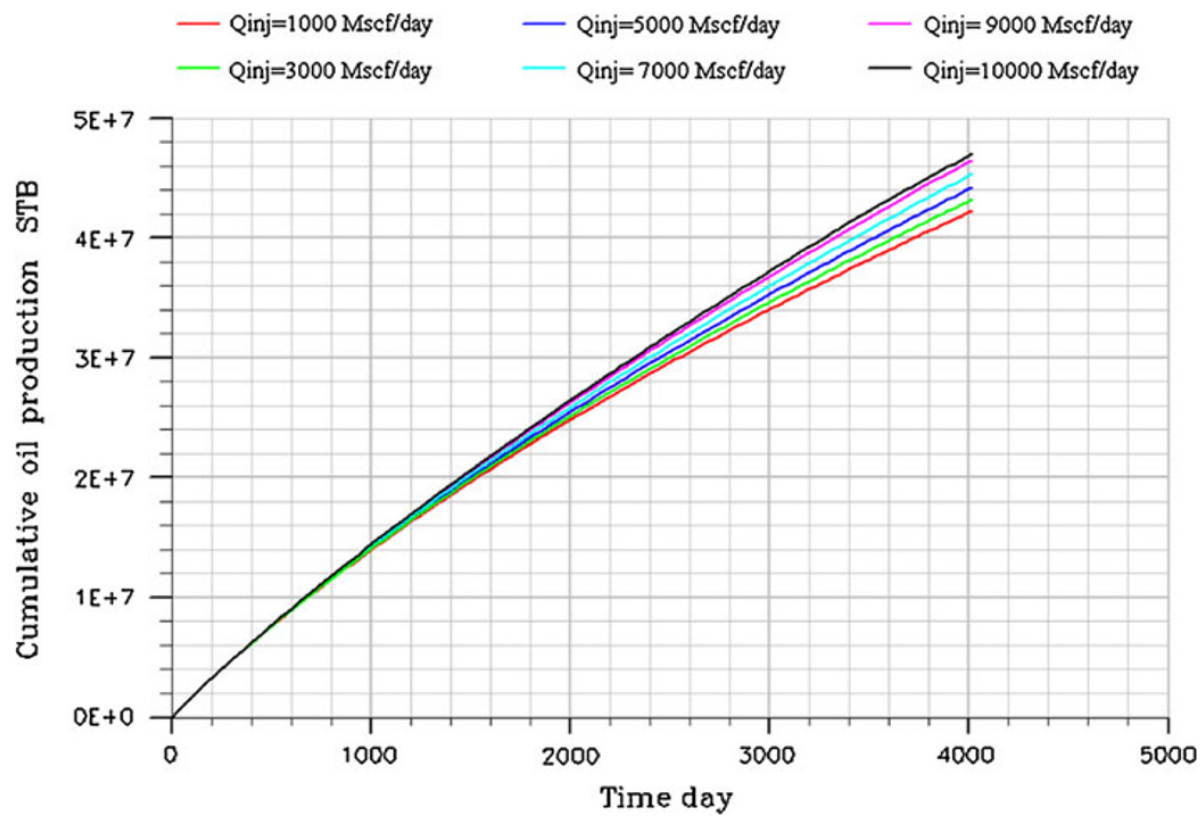

inner and border points are presented to study; therefore, it traps the non-linear effect in the system, and presents useful information for optimizing the development plan of Dena reservoir.

Using one variable at a time design, it can be observed that Pia, Va, BHPinj and MULTFLT parameters have the least effect on Dena reservoir development plan. On the other hand, MULTPV, MULTZ, Qinj and SGCR parameters have major effect on the development and future production of this reservoir.

Acknowledgments This research was supported by Department of Petroleum Engineering, Amirkabir University of Technology of Iran.

Open Access This article is distributed under the terms of the Creative Commons Attribution License which permits any use, distribution, and reproduction in any medium, provided the original author(s) and the source are credited.

\section{Appendix: effect of uncertain parameters on COP}

Substituting the COP values presented in Table 3, which have been obtained by simulator, into the following equations, we can calculate the effect of uncertain parameters on COP.

\section{Effect of uncertain parameter on COP $=\frac{\text { main effect of uncertain parameter on } \mathrm{COP}}{\sum \mid \text { main effect of uncertain parameter on } \mathrm{COP} \mid}$,}

where

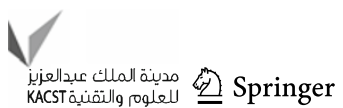

\footnotetext{
Main effect of uncertain parameter on COP

$=\mathrm{COP}_{(\text {maximum value of uncertain parameter })}$

$-\mathrm{COP}_{(\text {minimum value of uncertain parameter) }}$
}

\section{References}

Damsleth E, Hage A, Volden R (1991) Maximum information at minimum cost: a North Sea Field Development Study with an Experimental Design. In: Paper SPE 23139 presented at the SPE offshore Europe conference held in Aberdeen, 3-6 September 1991

Dejean JP, Blanc G (1999) Managing uncertainties on production predictions using integrated statistical methods. In: Paper SPE 56696 presented at the SPE annual technical conference and exhibition held in Houston, Texas, 3-6 October 1999

Eclipse (2005) Technical Description. Schlumberger, New York

Fetel E, Caumon G (2008) Reservoir flow uncertainty assessment using response surface constrained by secondary information. J Petrol Sci Eng 60:170-182

Kabir CS, Chawathe A, Jenkins SD, Olayomi AJ, Aigbe C, Faparusi DB (2002) Developing New Fields Using Probabilistic Reservoir Forecasting. In: Paper SPE 77564 presented at the SPE annual technical conference and exhibition held in San Antonio, Texas, 29 September-2 October 2002

Kloosterman HJ, Herring TR, Tull SJ, Van der Lee JW (2007) From test tube to value generator: effective use of Experimental Design for uncertainty analysis and development concept optimisation. In: Paper SPE 106893 presented at the SPE Europec/EAGE annual conference and exhibition held in London, United Kingdom, 11-14 June 2007

Kloosterman HJ, Herring TR, Tull SJ, Van der Lee JW (2008) Seeking the ultimate field development using the well maturity index. In: Paper SPE 113280 presented at the SPE Europec/EAGE annual conference and exhibition held in Rome, Italy, 9-12 June 2008

Moeinikia F, Alizadeh N (2011) Predicting the performance of an oil reservoir under immiscible gas injection by artificial neural network. Dissertation, Amirkabir University of Technology 
Spokes JJ, Ovuede MA, Ginger EP, Narahara GM, Haga O, Gontijo J (2004) Application of Experimental Design in selecting a development plan for the agbami field. In: Paper OTC 16994 presented at the offshore technology conference held in Houston, Texas, USA, 3-6 May 2004

Steppan DD, Werner J, Yeater RP (1998) Essential regression and Experimental Design for chemists and engineers. http://www. jowerner.homepage.t-online.de

Tabari K (2010) Impact of the sensitivity study and risk analysis on development of a large carbonate oil reservoir in Persian Gulf. World Appl Sci J 8(6):736-749

Venkataraman R (2000) Application of the method of Experimental Design to quantify uncertainty in production profiles. In: Paper
SPE 59422 presented at the SPE Asia Pacific conference on integrated modeling for asset management held in Yokohama, Japan, 25-26 April 2000

White CD, Royer SA (2003) Experimental Design as a framework for reservoir studies. In: Paper SPE 79676 presented at the SPE reservoir simulation symposium held in Houston, Texas, USA, 3-5 February 2003

Zhang J, Delshad M, Sepehrnoori K (2007) Development of a framework for optimization of reservoir simulation studies. J Petrol Sci Eng 59:135-146 